\title{
Analysing Single Photographic Images using Shape-From-Shading to Quantify Human Breast Profiles
}

\author{
Harvey MITCHELL* \\ Conjoint Fellow, School of Engineering, University of Newcastle, Newcastle, NSW, Australia \\ DOI: $10.15221 / 17.154$ http://dx.doi.org/10.15221/17.154
}

\begin{abstract}
Inspections of the three-dimensional shape of the human breast for various medical and para-medical purposes may be undertaken by visual observation of the breasts or of photographs of them, or by laser scanner measurement. It is proposed here that useful quantitative breast shape information may also be deduced by numerical analysis of a single digital photograph of the breast, using the principle of extracting an object's shape from image shading.

Shape-from-shading (SfS) is a method of deducing shape from the reflectance levels in a single photograph of an object. If an object has smooth physical texture and if it has light even colouring, the SfS technique is a possible means of obtaining the three-dimensional surface shape. SfS is appealing because of its one advantage: simplicity. SfS needs no special equipment, and involves the numerical analysis of a single digital photographic image, which is discussed in this paper.

Although SfS is restricted to objects of invariant colour texture and simple physical texture, it is suggested that those drawbacks may be tolerated for the measurement of some external surfaces of the human body, i) because the human skin surface colour is usually plain, ii) if surfaces are not too convoluted, and perhaps even iii) where some prior surface shape information can be assumed. It is envisaged that these three conditions may occur along certain profiles across the breasts. Along those profiles, a triflingly simple mathematical solution to SfS is available. An approach which is found to be workable in cases of measurement of breast-like shapes is proposed, and some preliminary and exploratory but informative tests of the technique are reported, with some accuracy tests of the technique on known shape objects and preliminary breast profile measurements. Motivation for further testing and development depends on its prospects for medical use in breast measurement.
\end{abstract}

Keywords: shape-from-shading, digital imagery, breast measurement

\section{Introduction}

If an object has a smooth physical texture, meaning that it is free from discontinuities, and if it is also of light even colouring, the technique of shape-from-shading (SfS) is an appealing means of seeking the object's three-dimensional surface shape. SfS, which involves deducing shape from only the reflectance levels in a photographic image of an object, is attractive because of its simplicity. SfS involves nothing more than the numerical analysis of a single digital image of the object of interest. (Multiple images may be used, in what is often called "photometric stereo" but the simpler case of the single image is being pursued here.)

Because measurement by SfS is limited to objects with specific physical and optical textures, its use is impracticable on many surfaces. As well, the SfS method suffers from another major disadvantage when analysing a single two-dimensional image: normally, there is an infinite number of solutions for the three-dimensional object shape. A solution is then only possible if some constraints are devised, which means having some prior knowledge of surface shape or making some assumptions about the three-dimensional characteristics of the object.

Despite these complications, the use of SfS for measurements of the three-dimensional shape of some areas of the human body may be viable, where the human skin surface colour is plain and where the body surfaces are not too convoluted. In particular, female breasts can generally be assumed to be smooth and essentially of an advantageous convex shape, although having the complications of the areola region and often a discontinuity of a skin fold on the lower side.

Two novel approaches are investigated in this work in an attempt to overcome the difficulties. Firstly, it is hypothesised that along certain lines over the female breasts, measurement of profiles is feasible. This is seen as a means of overcoming the problem of an infinite number of solutions. Secondly, profiles along the breast have a smoothness and even grade which suggests that the fitting of mathematical functions may be a viable means of coping with surface discontinuities, especially over the areola.

\footnotetext{
* harvey.mitchell@newcastle.edu.au; +61- 249 469298; www.newcastle.edu.au
} 
An approach to SfS which uses these two propositions together for the measurement of breast profiles is outlined here. with tests of the technique on geometrically shaped objects and some uncorroborated tests on a human breast. The work assumes a demand for breast shape measurement of various kinds.

\section{Principles of SfS}

\subsection{Conventional SfS}

The principle of SfS is that, if the object of interest is illuminated by a single light source (as distinct from general ambient lighting), then, at any pixel on a digital photographic image of that object, the image intensity value (i.e. grey-level or reflectance) is related by a known mathematical model to the surface gradient of that small element of the surface of the object. The goal of SfS is normally the determination of the surface gradients across the object from such an image, so that the three-dimensional surface shape can be deduced by numerical summation of the surface gradients. SfS theory mathematically relates the image reflectance at a pixel to the two gradients of the true surface at that pixel. The theory is easily uncovered via the usual searches.

The major predicament with the SfS method is that two gradients are needed to define the direction of the surface element at any one pixel, but only one reflectance value is available at each pixel, so that for any pixel there is an infinite number of solutions for the two gradients given the one reflectance. A solution seems to be apparent if an assumption can be made about the surface, e.g. where some prior surface shape information can be assumed, typically permitting the adoption of some surface model which relates the two gradients at one pixel to those at other nearby pixels. But the solution to the equations obtained in that way is only correct if the chosen constraint is perfectly valid. The constraining surface model may be provided only by a surface which is so well known that measurement is not beneficial!

This work looks at two approaches which may assist the SfS dilemma, both with a simplicity which matches the basic SfS concept.

\subsection{The use of Radial Profiles}

This work follows, firstly, a direction which may in practice be effective in various cases of human body measurement, and particularly in human breast shape measurement. That is, if, along a segment of the object, the gradient in one direction is known to be zero, then it permits a simple determination of the gradient in the other direction. In fact, if the cross-slope is not exactly zero but is close to it, then the other gradient is only marginally affected. Moreover, the profile approach is made mathematically simple if that line is also radiating from the centre of the image. It seems reasonable to envisage that these conditions may occur along lines radiating from the highest point of a breast, if imaging can be organised so that the object's closest point to the camera is located at the centre of the image. The method has the advantage that, not only is a solution possible, but the required equations become quite trivial.

The surface gradients calculated from along the profiles can provide useful surface shape information, but, as well, the full surface shape may be calculated by numerical integration of the surface gradients.

\subsection{Function Fitting}

In addition to the use of profiles, this work uses the fact that the shape of the breast is almost invariably even and smooth, which suggests the possibility of fitting relatively simple mathematical functions along the profiles mentioned in Section 2.2. Such functions have been investigated as a means of interpolating across those areas (notably the areola), which are sources of discontinuity in either the physical surface or the surface colour. Importantly, the parameters of these functions may define the surface sufficiently for some studies of the of breast shape.

\section{Laboratory Tests}

\subsection{Plane Surfaces}

Tests of the validity of the proposed mathematical model for SfS measurement of axial profiles have been undertaken using plane surfaces. Plane objects have the advantage that the assessment of the accuracy of results is straightforward. In each case, the plane has been imaged so that its gradient lies in either the $x$-direction (assumed to be in the direction of the rows of pixels) or the $y$-direction (assumed to be aligned with the image columns), so that it satisfies the requirement that the gradients in the crossdirection is known to be zero.

In the example related here, a plain painted wall was imaged from a distance of a metre using a Lumix Panasonic DMC-ZR1 camera. The wall was at an angle of $30^{\circ}$ to the plane of the camera's sensor, an angle given by an ordinary protractor, seen as an adequate method because the existence of any angle 
between the lens axis and the camera body was not known anyway. The separation between the camera's flash and the lens on this camera was regarded as insignificant at this object distance. The original colour image, at 4000 pixel columns $\times 3000$ pixel rows, was transformed to a monochrome image (to impart a single grey-level value to each pixel) and reduced in size to 144 columns $x 108$ rows (simply to bring the file to a convenient and manageable array size for subsequent analysis via a spreadsheet prepared by the writer) using proprietary image-manipulation software (PaintShop Pro X6 software (Version 16.2.0.20), from Corel Corporation, 1600 Carling Avenue, Ottawa, Ontario, K1Z 8R7, Canada). The camera focal distance, given by a calibration procedure, was 135 pixels (for the reduced image size).

Figure 1 shows the reflectance levels along the x-axis (taken to be row number 54 of the 108 rows). It is important to recognise that the reflectance levels do not immediately suggest that the object is plane. Figure 2 shows the deduced angles of the surface relative to the sensor. The points excluded from the orange line are a series of 12 points which all have the same integer value of the reflectance, 139 . The pixels up to an unexplained point of inflection at pixel $25(x=-51.5)$, seen in Figure 1, have simply been excluded from the calculations. The average surface slope angle of the surface elements relative to the plane of the sensor over 123 pixels along the sensor's $x$-axis is $31.6^{\circ}$, with a standard deviation of $\pm 1.1^{\circ}$.

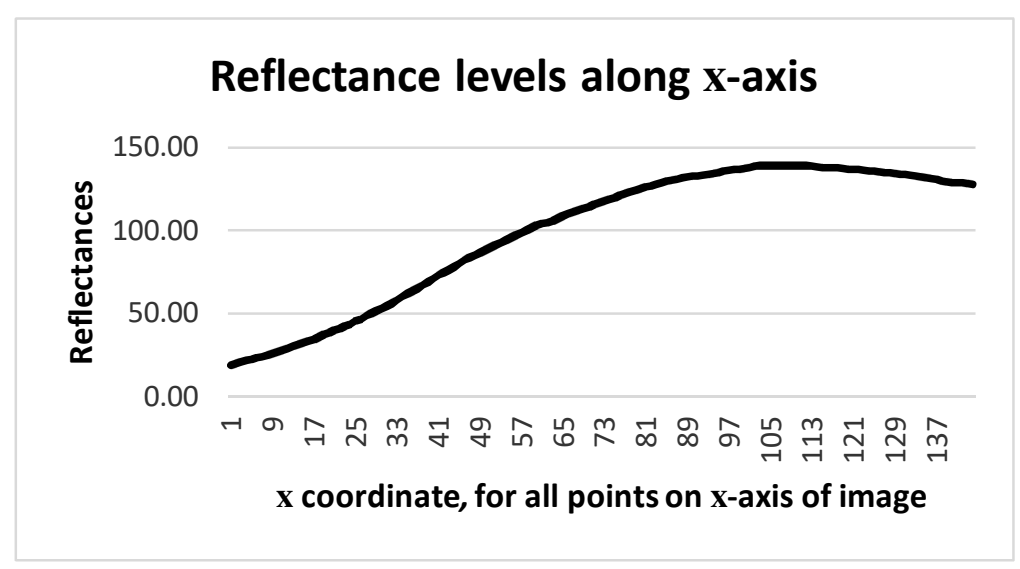

Fig. 1. Reflectance levels along $x$-axis of an image of a plane surface which is at an angle of $30^{\circ}$ to the camera's sensor. Figure by Microsoft Excel.

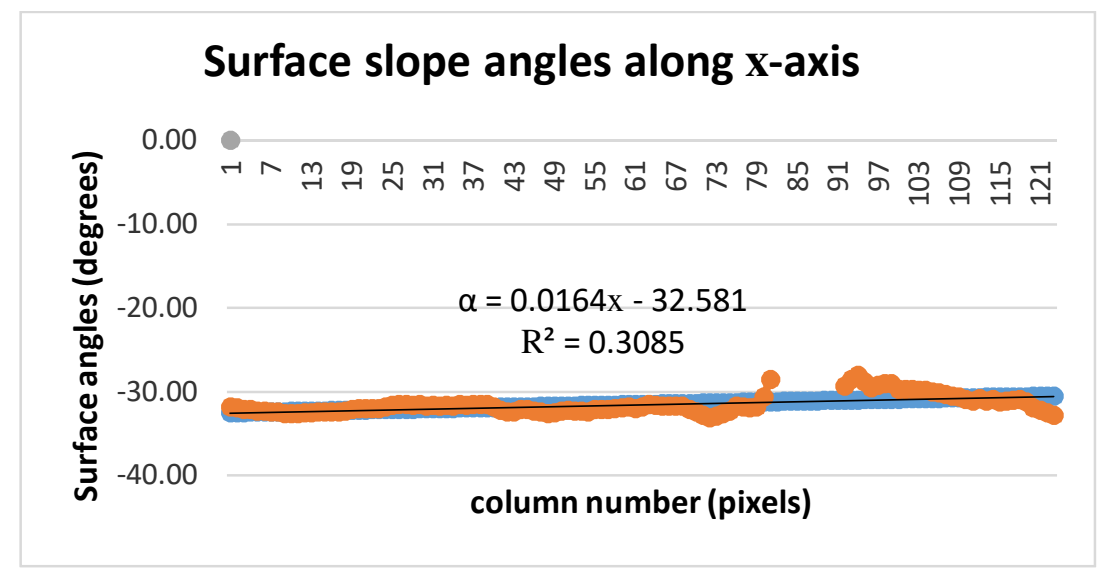

Fig. 2. The deduced angles between the wall surface and the plane of the sensor, along the $x$-axis of an image, and the straight-line fit (with the best-fitting equation shown). A number of points at the start of the line and in the

vicinity of the maximum reflectance have been omitted (see text). The straight line shows where the missing surface angles have been interpolated. Figure by Microsoft Excel.

The equation of the straight line fitted to the surface slope angles in degrees in Figure 2 is

$$
\alpha=0.0164 x-32.58
$$

where $\alpha$ is the surface angle in the $x$-direction, with the $\mathrm{R}^{2}$ statistic having a value of 0.31 . The existence of a small coefficient of $x$ in Equation (1) above suggests the wall shape line is a parabola but with a small vertex angle.

The SfS measurement of the plane object was regarded as being acceptable for the purposes at this stage of the investigation. 


\subsection{Cylinder}

Further tests of the validity of the mathematical model for SfS profiling have also been undertaken with a small metal cylinder, positioned vertically, as in Figure 3. The goal was to see whether it was possible to recover the circular shape along a profile of the cylinder's cross-section. The cylinder, of $107 \mathrm{~mm}$ diameter, was covered with plain white paper to give it the necessary light and even colour. An image was taken with an Apple iPhone 4S mobile phone camera, with illumination by the camera's flash. Mobile phone cameras have the distinct advantage that the flash is located very close to the lens (but that does not ensure their parallel alignment). The camera was about $200 \mathrm{~mm}$ from the closest point of the cylinder, and oriented so that the flash was directed at centre of cylinder as closely as possible. The surface gradients along the long axis of the cylinder should be close to zero, satisfying the requirements that gradients occur in the $\mathrm{x}$-direction, making possible the determination of gradients along the $\mathrm{x}$-axis by the specific SfS profiling method being proposed here.

The original JPEG colour image, of 1936 columns x 2592 rows (according to the image's Exchangeable Image File Format, EXIF), was reduced to a monochrome image of 108 columns $\times 144$ rows, as used in the case of the plane object discussed above. Calculations were based on a camera focussing distance of 120 pixels, obtained by a calibration process and applicable to an image size of $144 \times 108$ pixels. Reflectance values were smoothed by taking simple averages of three consecutive values.

Figure 3 shows the raw image, with the reflectance levels contoured to emphasise (as does Figure 1) that the reflections levels in the raw image do not depict the shape of the object.

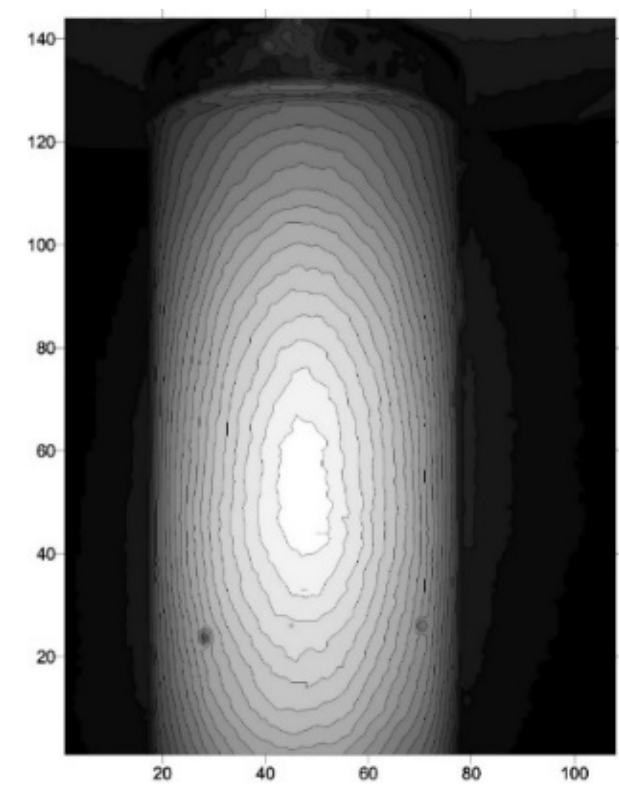

Fig. 3. Flash photograph image of paper covered cylinder, with the reflectance levels contoured to emphasise that the reflectance levels do not directly depict the object shape. The contoured interval is 10 units of reflectance, the maximum being 214 . The units on the axes are pixels.

Figure 4 shows the reflectance values across the middle row of the image of the cylinder in Figure 3 , while Figure 5 shows the angles of the surface slope (in radians) over the cylinder's cross-section, deduced from the smoothed reflectances shown in Figure 3 using the proposed SfS profiling process. The units on the axes are pixels. Pixels in the image background and beyond the cylinder are excluded, so the figure is based on the remaining 64 pixels only, of 108 pixels in the row. It is obvious that the slopes tend to a straight line, and a best fit second-order line is shown, to indicate that the coefficient of $x^{2}$ is only 0.00001 . The surface slope angles shown in Figure 5 were converted to surface gradients by summing the trigonometric tangent of the surface slope angles, providing the surface shape shown in Figure 6. A second-order polynomial fitted to the shape has the equation shown on that figure. Two marks on the circumference to provide scale, were $65 \mathrm{~mm}$ apart and on the image 43 pixels apart, giving a scale of $1.5 \mathrm{~mm} /$ pixel.

Assessment of the accuracy of the results reveals the following. The radius of curvature at the highest point of the parabola where $\mathrm{x}$ is small, close to the centre of the image, is 35.5 pixels. At an image scale or $1.5 \mathrm{~mm}$ per pixel, the radius is $53 \mathrm{~mm}$, satisfactorily matching the measured diameter of 107 $\mathrm{mm}$. This acceptable outcome for the cylinder is assumed to imply the possibility of measuring horizontal breast profiles by a similar process. 


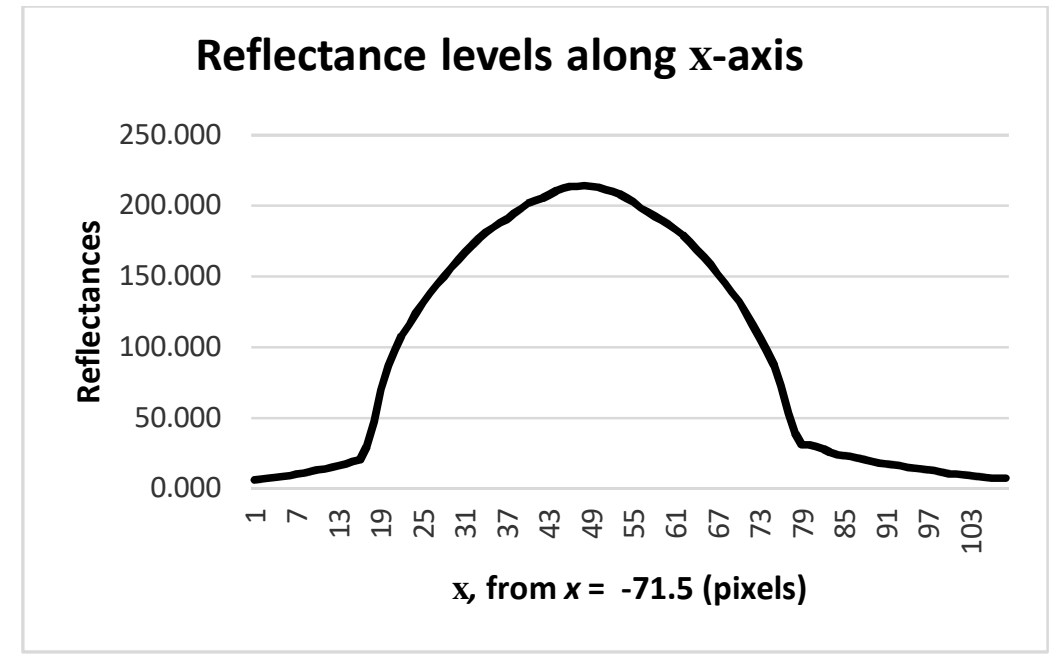

Figure 4: The reflectance values across the full middle row of the image of the cylinder at Figure 3. Figure by Microsoft Excel.

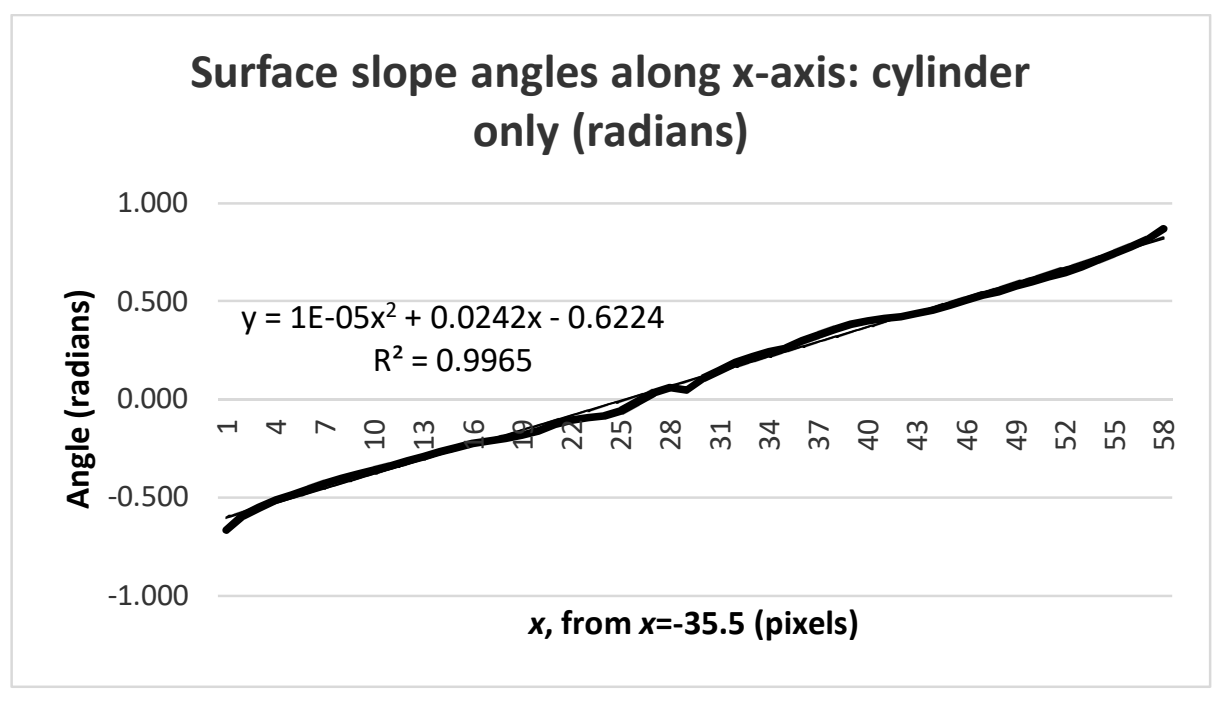

Fig. 5. The deduced surface slope on the cross-section of the cylinder only, i.e. pixels beyond the cylinder are excluded, so the figure is based on the remaining 64 pixels; see Figure 4. Figure by Microsoft Excel.

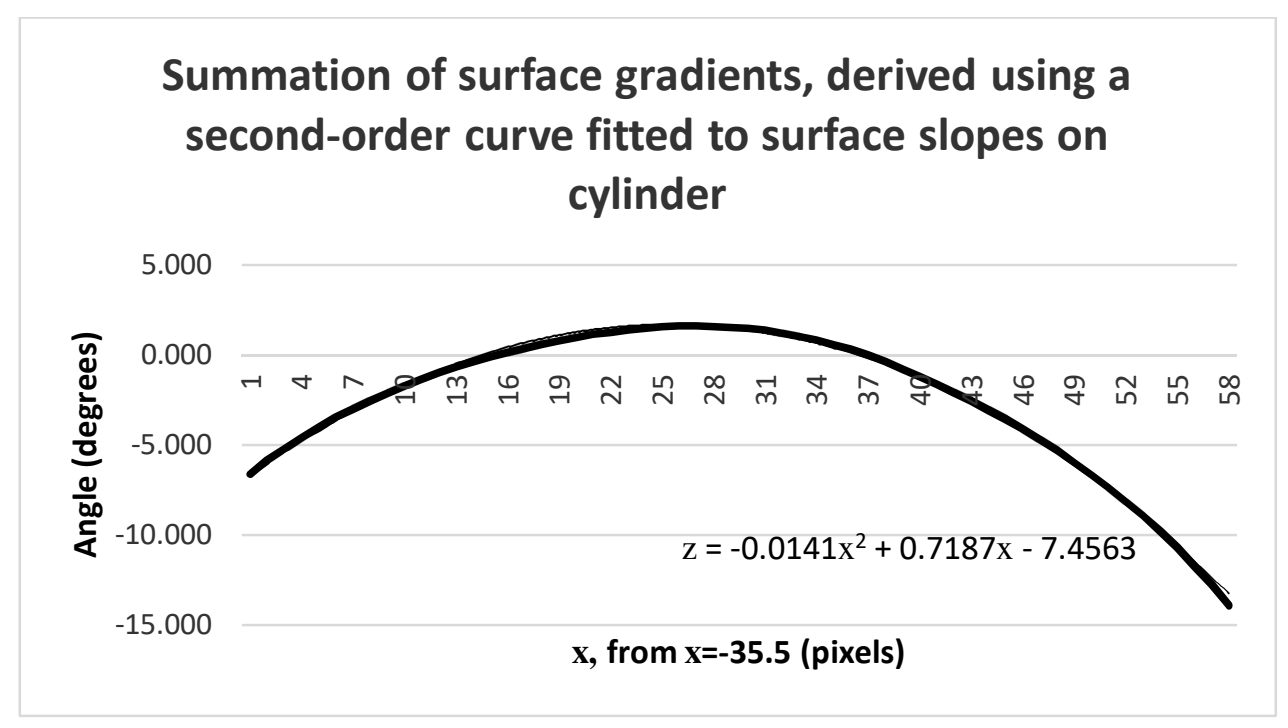

Fig. 6. Surface shape given by the summation of the tangents of the surface slope angles, which were derived using a straight line fitted to surface slopes shown in Figure 5. Figure by Microsoft Excel. 


\subsection{Horizontal Profile on Breast}

The technique used above has been developed with the specific goal of applying it to human female breast measurement. It is appreciated that certain practical challenges must be faced before the technique is viable. Despite some reservations, exploratory calculations of feasibility have been undertaken on just two human female breast images. In neither case has the result been verified, because of the difficulty of obtaining confirmation by an alternative measurement technique.

In the one example reported here, a Flexiscope brand Piccolo intra-oral dental camera, providing colour imagery at a size of $768 \times 576$ pixels was used for imaging a left breast. The camera had the advantages of being of high quality but moreover of having the lighting provided by two elongated sources parallel to the y-axis, each side of and therefore very close to the lens. The image was reduced to a monochrome image with 144 columns and 108 rows, and the focal distance by calibration was 125 pixels for an image of that size.

Figure 7 shows the reflectances occurring a) over the entire image and b) in a profile along the middle row of the image, after being smoothed by taking simple averages of three consecutive values, as was done for the cylinder. The image covers the breast and extends into the left arm, revealed as low-level reflectances on the right-hand side. The calculations referred to below are based on pixels in columns 10-111 only. It is to be noted that the areola is central to the image, which had been positioned appropriately for the imaging, according to a requirement suggested above, and covers pixels 38 to 87 . For privacy, the raw breast image is not shown.

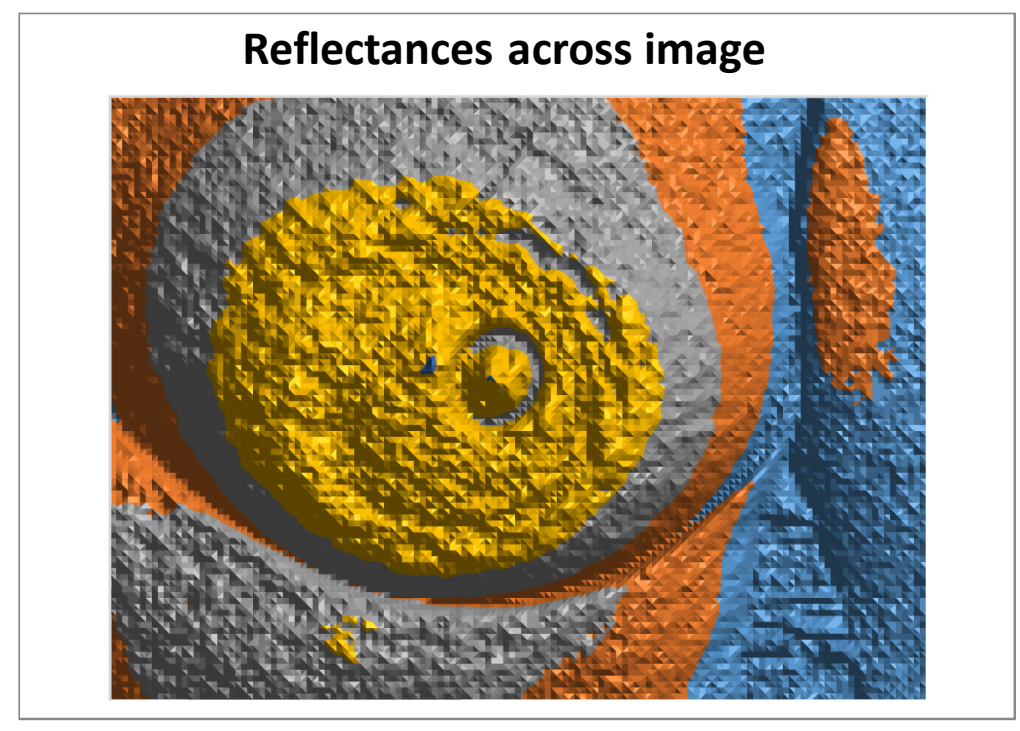

a)

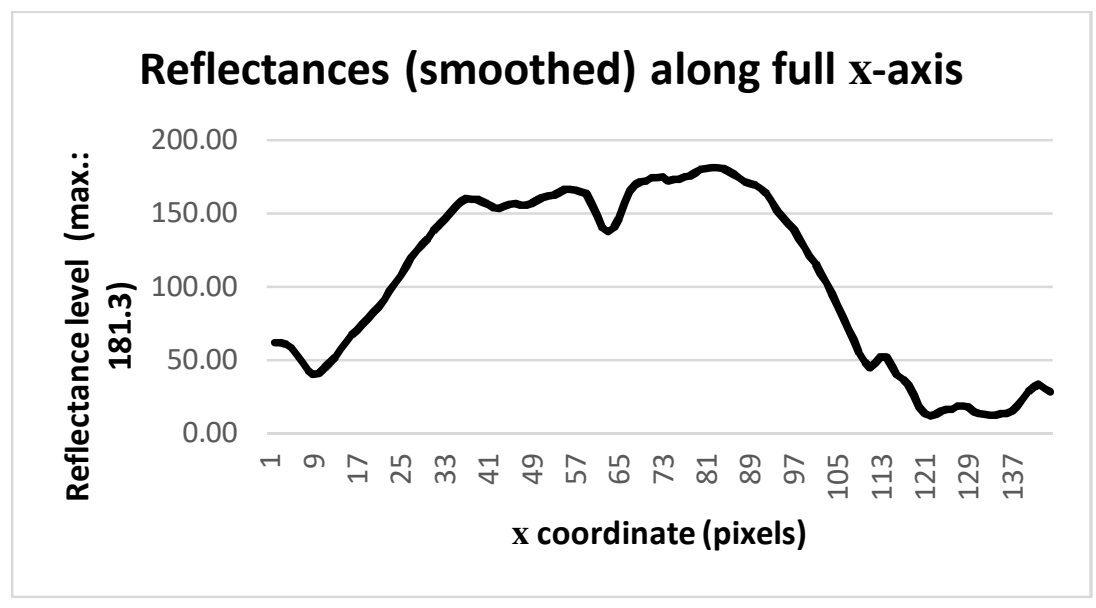

b)

Fig. 7. Reflectance levels along row 54 of the image of a breast shown at a). The occurrence of the areola region from about pixels 38 to 87 is apparent. The change in grade on the lower left occurs as the image reaches a discontinuity at the edge of the torso and includes some of the subject's left arm. Figures by Microsoft Excel. 


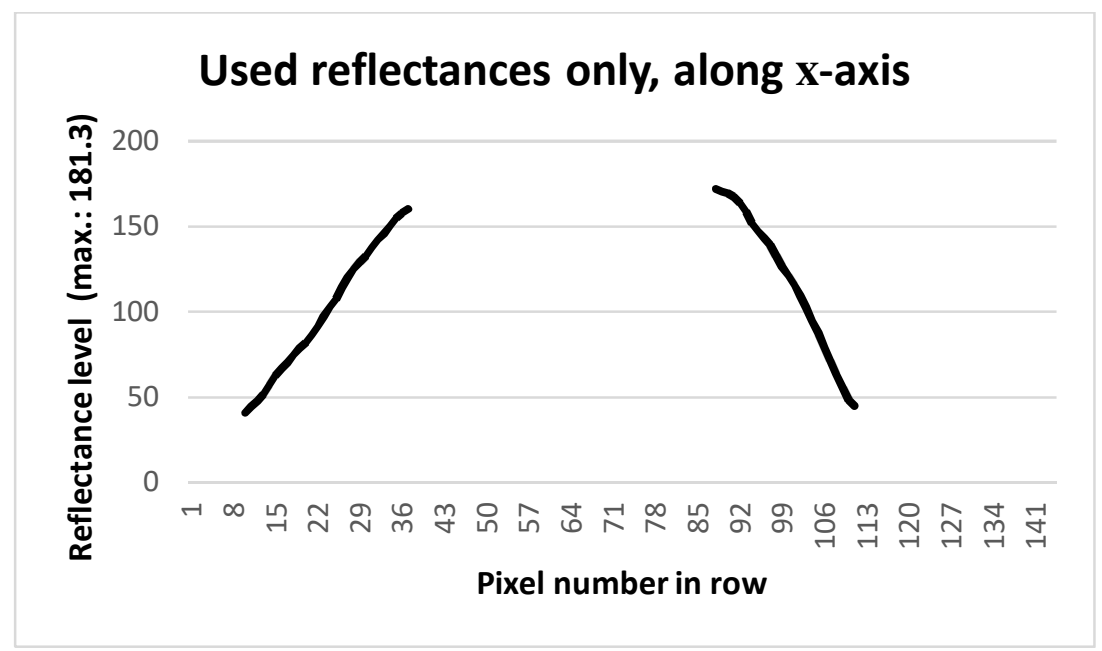

Fig. 8. Reflectance levels along row 54 of an image of a breast, showing only the pixels used to define the normal breast, excluding the areola region estimated to be from pixels number 38 to 87 . Figure by Microsoft Excel.

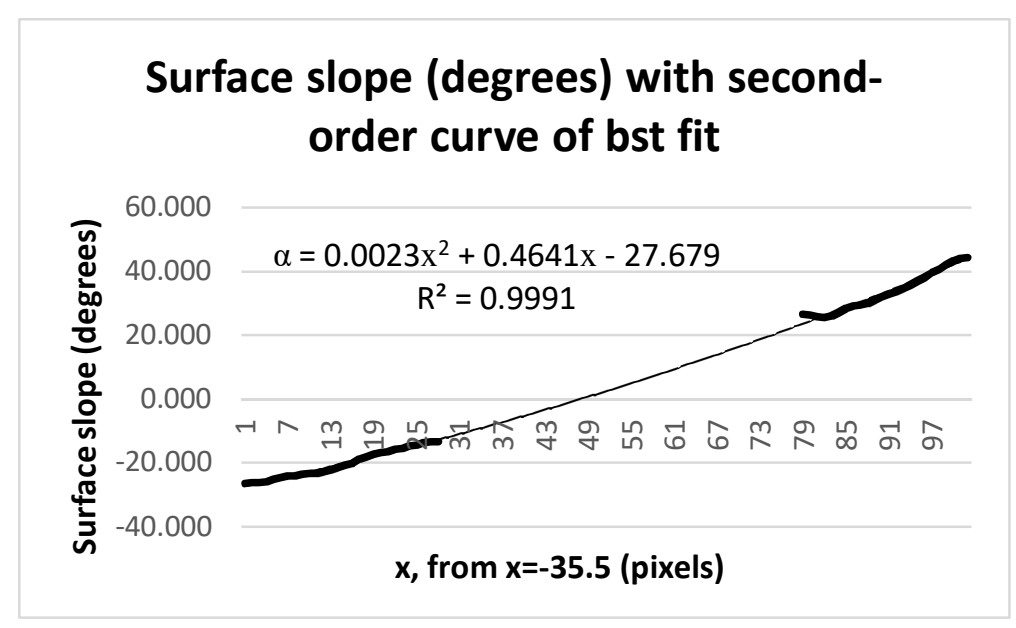

Fig. 9: Surface gradients, as given along row 54, calculated for only those pixels shown in Figure 8. Figure by Microsoft Excel.

Figure 8 shows the reduced number of reflectances that were used in the SfS profile.

Figure 9 shows the deduced surface slope angles along the profile of points used in Figure 8.

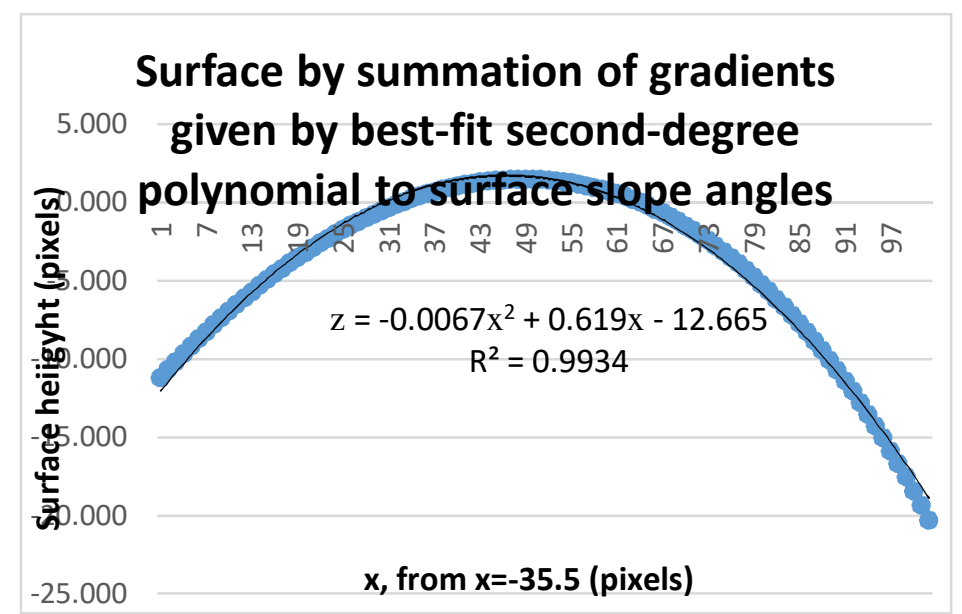

Fig. 10. The breast surface shape as given by a summation (outwards from the centre) of the gradients (tangent of the surface angles) derived from the best-fit curve $\left(\mathrm{y}=0.0023 x^{2}+0.4641 x-27.679\right)$, shown in Figure 9. The summation covers only the pixels which are seen to be in the breast area, i.e. between 10 and 111 (see the breast limits in Figure 7). The curve interpolates over that area which is areola. Figure by Microsoft Excel. 
The almost linear nature of the segments together suggests that the surface obtained by summation of all the gradients may tend to be a simple continuous surface, which can be represented by a simple second-degree polynomial. The gradients increase in value away from the centre of the breast, as expected, and they change sign at about the centre of the breast. Also shown is the trend-line which suggest that there is a simple relationship between the two segments of gradients. The best-fit polynomial derived from the surface summation is shown in Figure 10. A second-degree curve was fitted to the surface angles across the usable parts of the breast. It was not possible for the scale of the image to be calculated.

Figure 11 is similar to Figure 8 , but it is extended to include pixels beyond the breast. It suggests that small anomalies of interest in the breast shape may be revealed as deviations from the straight lines.

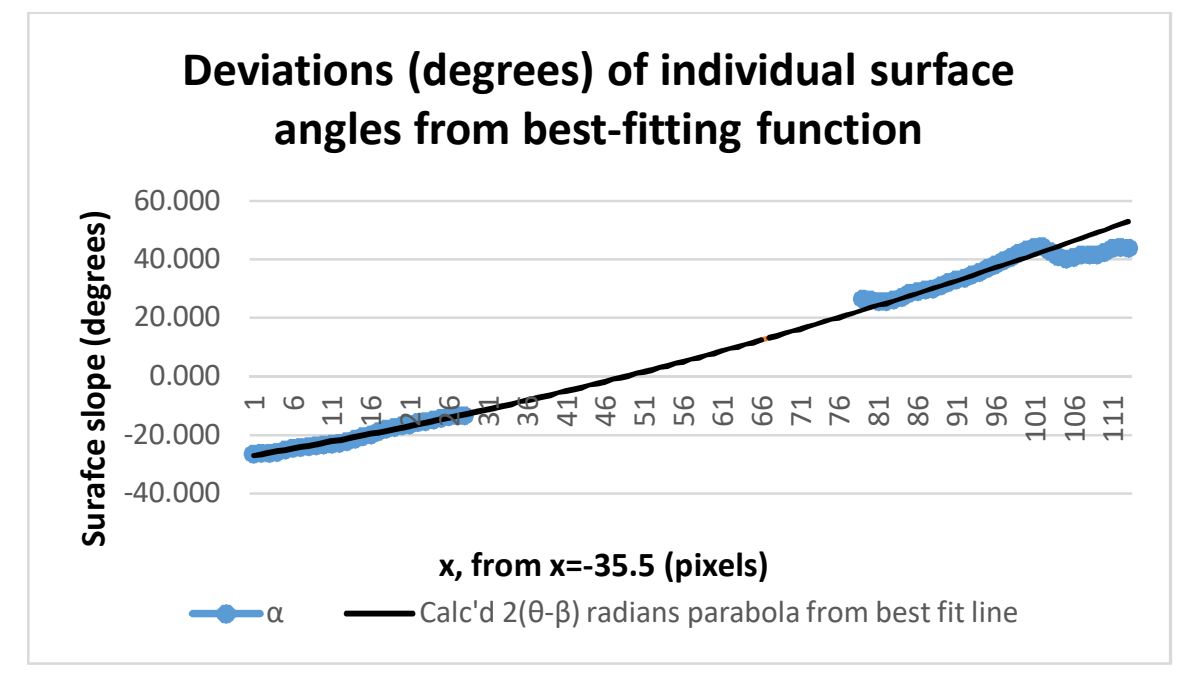

Fig. 11: Surface slope (degrees) with as in Figure 9, but with pixels beyond the columns used in Figure 9, revealing the surface beyond the breast. Figure by Microsoft Excel.

\subsection{Tests on Full Three-Dimensional Surface of a Breast}

Determination of the surface angles over the complete breast can be undertaken if it is valid to assume that, along radial lines, the surface gradients are overwhelmingly radial. The subsequent step, computing surface heights by summations in the radial directions, is made difficult when the image is supplied as a rectangular array, but that step may not be crucial if it is feasible to utilise the surface slope angles, rather than the surface itself. Calculations of the radial surface angles have been undertaken on the full breast used in the profiling example above, showing smoothly varying angles, but the results are difficult to depict, and a diagram is not included here. However, the profile obtained in the vertical or y-direction of a breast is shown in Figure 12. The circumstances were similar to that case covered in the sub-section above. A mathematical function was again fitted to the profile, but in this case a third-order polynomial (as shown) was fitted to the shape.

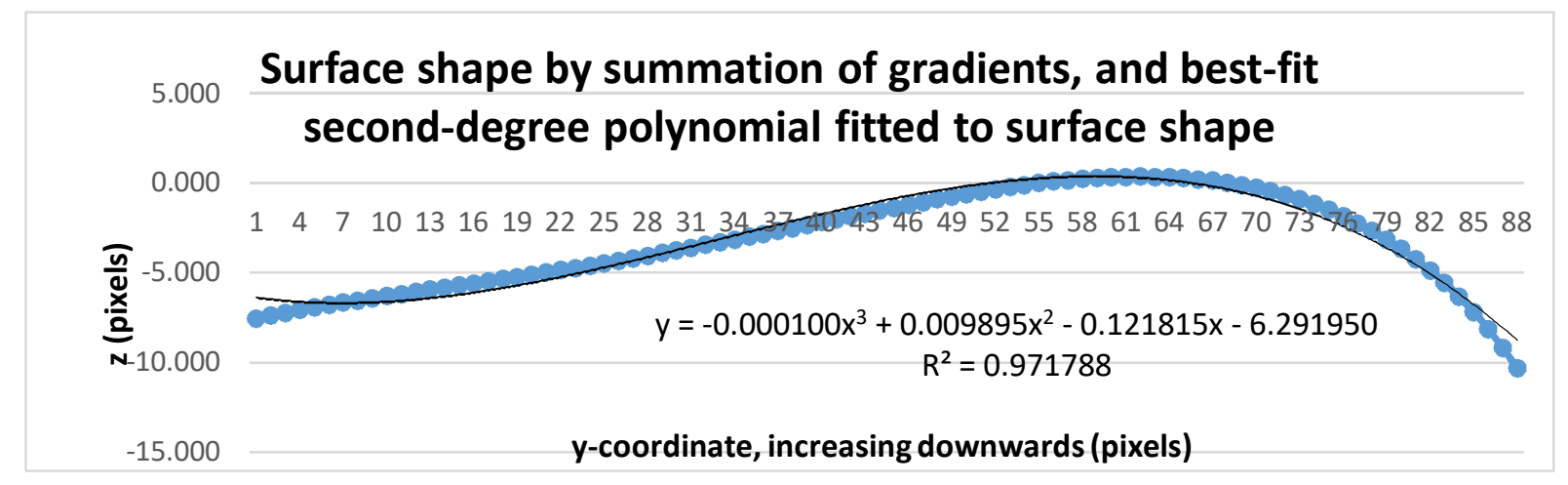

Fig. 12. The breast surface shape as given by a summation of the gradients (tangent of the surface angles) for a vertical profile. The summation covers a limited number of pixels which are seen to be in the breast area.

The curve covers the area which is in fact the areola. Figure by Microsoft Excel. 


\subsection{Discussion of Results}

Although the accuracy of the breast measurements needs to be confirmed, results so far are encouraging. Some outcomes are unexpected: the level of effectiveness of the mathematical function fitting was not anticipated. The fact that a simple mathematical function, barely different from a straight line, has been fitted to the two separated parts of the breast, as in Figure 9, was unexpected and revealing. A single uncomplicated function, perhaps no more than linear, may model the breast profile's surface angles from one side to the other.

The outcomes suggest the possibility that using the SfS results presented as surface angles may be easier and more useful that converting the angles to a surface shape (a process which can accumulate noise and errors).

The number and scope of the tests which have been undertaken so far is limited. More than anything else, the accuracy of the SfS method used specifically for breast measurement under various conditions and with different equipment has not been established. The SfS profiling theory assumes that that along the radial lines of the image there is no transverse gradient on the object. On the female breast, that may or may not be precisely true, and it deserves to be ascertained.

Further testing is needed to extend and diversify the cases studies, with investigations into the repercussions of such matters as: image characteristics (especially noise levels), effects of converting an original image from colour to monochrome and its re-sizing; the existence of and the effect of any misalignments between the flash axis and the camera lens axis; the nature of the illumination provided by a flash; the optimum number of pixels; and so on. Finally, it should be possible to determine the best mathematical functions which satisfactorily represent the breasts, either as profiles or in threedimensions.

It is apparent that the steeper regions of the cylinder and the breast are not covered by the this SfS method, perhaps because of the use of the flash lighting.

Should implementation of the technique be seen as worthwhile the first priority would be to transform the current experimental system into a feasible demonstration system. The SfS method's main advantages has been described as simplicity of data collection, which requires neither special tools nor special skills, and it follows that a working demonstration system would need to be inexpensive.

\section{Discussion and Conclusions}

The work concentrates on the investigation of the concept, not on its implementation. Preliminary results suggest that the radial breast profile does appear to be measurable by a simple SfS algorithm. More than that, the particular radial breast profile in the direction across the chest appears to be modellable with a mathematical function of up to no more than third order, while a model of the shape in other directions too, seems feasible.

Some difficulties in the method are foreseen. The image processing can be assumed to be successful only on bare human skin which has an even physical texture and light optical texture, free from colour variations. Testing has not been carried out on darker or varying colour skin, due to such things as pigment, tanning or tattoos. The option of wearing of tight, light coloured clothing has not been tried. The SfS method's simplicity, including the feasibility of using a simple mobile phone camera, and its quantitative output should make it superior to human examination of either imagery or the patients themselves, and may make it useful for various medical and para-medical purposes. SfS is not being proposed here as an alternative to laser scanning, which provides data which is clearly more accurate and comprehensive and more easily comprehended than the SfS method does. On the other hand, scanners are a resource which is relatively expensive and not necessarily as easily made available or operated, but they can be worth accessing in some situations. Other optical techniques, such as photogrammetric, structured light and moiré fringe methods, have also been proposed for breast measurement, but they do not appear to be in common use.

Motivation for further testing and development depends on its prospects for use. Breast measurement seems to be most suitable for measurement by the simple surface model based on the radial line assumption, i.e. that gradients are in the direction radial from the centre of an appropriately positioned camera and that mathematical functions can be fitted to the surface. Numerous questions arise about the demand for breast measurement, whether the appropriate shape information be deduced in practice from the measured profiles. No attempt has been made to isolate suitable applications of breast measurement, which are not an area of expertise of the writer, but the general existence of demand is apparent in the literature. 